\title{
Phlegmonous gastritis in an ankylosing spondyli- tis patient treated with infliximab
}

\author{
Bo Young Kim ${ }^{1}$ and Hyun-Sook Kim ${ }^{2}$
}

${ }^{1}$ Division of Rheumatology, Department of Internal Medicine, Gangneung Asan Hospital, University of Ulsan College of Medicine, Gangneung; 'Division of Rheumatology, Department of Internal Medicine, Soonchunhyang University College of Medicine, Seoul, Korea

Received: November 23, 2015

Revised : January 6, 2016

Accepted: January 13, 2016

\section{Correspondence to}

Hyun-Sook Kim, M.D.

Tel: +82-2-709-3061

Fax: +82-2-709-9554

E-mail: healthyra@schmc.ac.kr
A 51-year old man, with a 2-year history of ankylosing spondylitis (AS), was hospitalized for severe nausea and vomiting. On physical examination, his temperature was $37.5^{\circ} \mathrm{C}$ and his blood pressure was 138/86 $\mathrm{mmHg}$. His laboratory findings were as follows: white blood cell count, 15,200/ $\mathrm{mm}^{3}$ (neutrophils, 81.6\%); hemoglobin, 9.4 $\mathrm{g} / \mathrm{dL}$; platelet count, $400,000 / \mathrm{mm}^{3}$; erythrocyte sedimentation rate, $74 \mathrm{~mm} / \mathrm{hr}$; and C-reactive protein, $4.00 \mathrm{mg} / \mathrm{dL}$. He had been treated with infliximab beginning 6 months earlier and had last received it 2 weeks earlier. Abdominal computed tomography (CT) showed diffuse edematous submucosal wall thickening throughout the stomach and no specific findings in the colon (Fig. $1 \mathrm{~A}$ and 1B). A characteristic feature of phlegmonous gastritis is the isolated thick- ening of the gastric wall, most marked in the submucosa. Esophagogastroduodenoscopy showed edematous, reddish mucosal change, and multiple ulcerative lesions on the greater curvature of the stomach. Diffuse ulcerative lesions on the fundus of the stomach were also detected (Fig. 1C). Phlegmonous gastritis was diagnosed based on CT and endoscopy findings. No phlegmonous gastritis pathogen was identified from cultures of blood, gastric aspirates, or tissue. The patient was treated conservatively with fluid resuscitation, a proton pump inhibitor, and broad-spectrum antibiotics including vancomycin and piperacillin/tazobactam for 3 weeks. Within a few days, the patient was afebrile and able to eat.

Phlegmonous gastritis is a rare, rap-
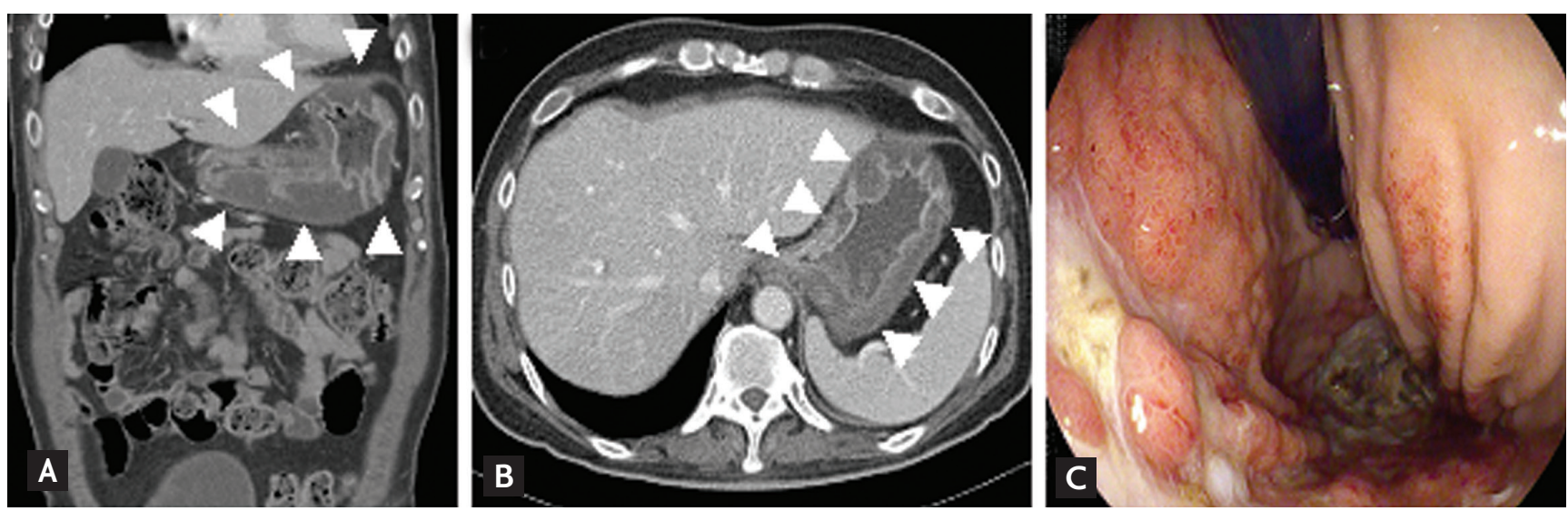

Figure 1. (A, B) Computed tomography showed diffuse edematous submucosal wall thickening throughout the stomach, but no specific findings in the colon. (C) Esophagogastroduodenoscopy showed edematous, reddish mucosal changes, and multiple ulcerative lesions on the greater curvature of the stomach. Diffuse ulcerative lesions on the fundus of the stomach were also detected. 
idly progressive, potentially fatal bacterial infection. Predisposing factors include mucosal injury, achlorhydria, and an immunocompromised state. Phlegmonous gastritis may follow a fulminating course involving early peripheral circulatory collapse or delayed gastric perforation. Sepsis and multiorgan failure are common. However, the rarity of this disease makes the diagnosis and choice of appropriate treatment difficult.
Early recognition and therapy are important for survival. We report a case of phlegmonous gastritis in AS that was diagnosed early and treated successfully with broad-spectrum antibiotics.

\section{Conflict of interest}

No potential conflict of interest relevant to this article was reported. 